\title{
États-Unis, Québec : le débat sur les droits d'inscription dans le supérieur
}

\section{Maroussia Raveaud}

\section{OpenEdition}

\section{Journals}

Édition électronique

URL : http://journals.openedition.org/ries/309

DOI : $10.4000 /$ ries.309

ISSN : 2261-4265

\section{Éditeur}

Centre international d'études pédagogiques

Édition imprimée

Date de publication : 1 avril 2008

Pagination : 17-20

ISBN : 978-2-85420-572-5

ISSN : $1254-4590$

\section{Référence électronique}

Maroussia Raveaud, «États-Unis, Québec : le débat sur les droits d'inscription dans le supérieur »,

Revue internationale d'éducation de Sèvres [En ligne], 47 | avril 2008, mis en ligne le 24 juin 2011 consulté le 30 avril 2019. URL : http://journals.openedition.org/ries/309 ; DOI : 10.4000/ries.309

Ce document a été généré automatiquement le 30 avril 2019.

(c) Tous droits réservés 


\title{
États-Unis, Québec : le débat sur les droits d'inscription dans le supérieur
}

\author{
Maroussia Raveaud
}

1 Les effets de la «massification » scolaire intervenue dans l'enseignement secondaire des pays industrialisés se font ressentir sur les effectifs de l'enseignement postsecondaire. Dans un contexte de concurrence économique mondialisée, les États se préoccupent d'élargir l'accès à l'enseignement supérieur, tout en renouvelant les formes de financement afin que l'augmentation des dépenses ne repose pas sur la seule puissance publique. En particulier, les étudiants de l'enseignement supérieur supportent une part croissante du coût de leurs études sous forme de droits d'inscription.

\section{Effort public, effort personnel de financement}

2 Les solutions retenues par les États varient très fortement : au Danemark, les étudiants ne paient pas de droits d'inscription et l'État finance entièrement les études. Aux États-Unis, pays de l'OCDE ayant la plus haute dépense par étudiant dans l'enseignement supérieur, la proportion du financement public est bien inférieure à la moyenne ${ }^{1}$ et les étudiants supportent des droits d'inscription trois fois plus élevés en moyenne que dans l'ensemble de l'OCDE (avec une variation de 1 à 8 autour de cette moyenne). Les droits moyens dans les établissements supérieurs publics sont de $\$ 6185$, un montant majoré de $\$ 10000$ pour les étudiants issus d'un autre état, et $\$ 23712$ pour les établissements privés ${ }^{2}$. Le fait que ces droits aient connu une augmentation plus rapide que le taux d'inflation a ceci de paradoxal que les subventions publiques aux États-Unis ont été accrues, délégitimant l'argument habituel qui consiste à justifier la hausse des droits par un manque de financement par ailleurs. Parmi les causes avancées: la volonté des établissements supérieurs d'accroître leur budget total pour devenir plus compétitifs sur un marché mondial, et les effets des restrictions budgétaires passées. De surcroît, les mesures de gel 
des droits d'inscription décidées par certains états peuvent conduire à des augmentations "préventives", comme en Géorgie où la garantie que les frais n'augmenteront pas pendant les quatre années d'études pour les nouveaux étudiants se traduit par des droits accrus de 15,5\% en 2007 alors même que les subventions ont progressé de plus de $10 \%$.

\section{Les aides aux étudiants}

3 L'augmentation des droits d'inscription ne se traduit pas nécessairement par un surcoût pour l'étudiant, et sa situation financière dépend largement des aides proposées. C'est ainsi que les États-Unis ont été classés quatrième selon un indice composite d'accessibilité à l'enseignement supérieur en 2005, juste devant le Canada, laissant la France huitième en dépit de droits d'inscription faibles ${ }^{3}$.

4 Si les droits aux États-Unis ont augmenté de plus de $6 \%$ en 2007-08, les aides aux étudiants évoluent dans les mêmes proportions, et l'État fédéral intervient de plus en plus pour financer des bourses sur critères sociaux (Pell grants) et des prêts subventionnés (avec les Stafford loans, le gouvernement paie les intérêts pendant la durée des études). À plus long terme toutefois, l'augmentation des aides $(+41 \%$ pour les bourses Pell en dix ans) est bien inférieure à l'évolution des droits, d'où la nécessité grandissante de recourir à des prêts privés. Les scandales judiciaires du printemps 2007 touchant les intermédiaires privés ont conduit à une loi votée en septembre 2007 (Student Loan Sunshine Act) qui vise à accroître les financements publics et à réduire le recours à ces intermédiaires. Des inquiétudes concernant les inégalités sociales dans l'accès à l'université ont par ailleurs conduit certaines universités privées, dont Harvard et Yale, à remplacer les prêts par des bourses accordées aux étudiants les plus démunis.

5 Harvard, suivie d'une trentaine d'universités privées, a étendu aux classes moyennes l'allègement des droits d'inscription: par une décision de décembre 2007, les étudiants dont les parents gagnent jusqu'à 180000 dollars par an verront leurs droits plafonnés à $10 \%$ de ces revenus. La presse souligne le risque de disparités entre la situation des étudiants des établissements d'élite et ceux des universités publiques ou d'autres établissements privés qui n'ont pas de fondations susceptibles de finances de telles aides.

\section{Des droits dissuasifs pour les plus défavorisés?}

6 Ces initiatives suggèrent l'inquiétude qui règne concernant l'accès à l'enseignement supérieur et les inégalités entre classes sociales. Aux États-Unis, le taux de participation éducative des 20-29 ans est inférieur à la moyenne de l'OCDE, avec des écarts significatifs de participation suivant le milieu social. La recherche est partagée sur les répercussions de l'augmentation des droits accrus et des perspectives d'endettement sur le choix d'entamer des études parmi les plus démunis ${ }^{4}$.

7 L'originalité du Québec en la matière a été soulignée par un débat récent. Contrairement aux provinces anglophones, le Québec avait fait le choix de droits réduits, gelés en 1994 à 1668 dollars canadiens (1 1845), ce qui rendait les études québécoises 3,5 fois moins chères qu'ailleurs dans le pays. La part du financement couverte par les droits de scolarité y était de $8,3 \%$ contre 20 à $25 \%$ dans les autres provinces canadiennes en 2006-075. Or le gouvernement du libéral Jean Charest a augmenté les droits de $50 \$(35,505)$ par semestre sur cinq ans à compter de la rentrée 2007, soit une augmentation de $30 \%$. Cette décision a 
divisé le parlement, les présidents d'université et les étudiants. Tandis que le président de la Conférence des principaux des universités du Québec y voit «une augmentation modeste et bien prévisible», un rapport de recherche indépendant suggère une spécificité québécoise : contrairement aux provinces anglophones, l'augmentation des droits conduirait au Québec à une chute dans la fréquentation universitaire, selon les projections de Valérie Vierstaete, une crainte étayée par l'expérience des années $1990^{6}$.

\section{Scénarios pour la contribution étudiante}

8 Un acteur très présent dans le débat québécois a été le syndicalisme étudiant. L'Association pour une solidarité syndicale étudiante (ASSE), de gauche, appelle à la gratuité d'un enseignement supérieur financé par un impôt sur le revenu plus progressif. La Fédération étudiante universitaire du Québec (FEUQ), principale fédération étudiante de la province, dénonce l'augmentation des frais, mais se penche parallèlement sur divers modèles consistant à différer le paiement jusqu'à ce que l'étudiant ait trouvé un emploi rémunérateur7. Le scénario du "remboursement proportionnel au revenu », proche des expériences britannique et australienne, revient à transformer les frais des études en dette individuelle remboursable une fois les études terminées. Un autre scénario, sans précédent connu, consisterait en un «impôt postuniversitaire », dépendant du revenu et non pas du coût de sa propre formation, de sorte que les diplômés financent tout au long de leur vie active l'ensemble du système universitaire. Le syndicat souligne toutefois le risque de désengagement de l'État dans le financement des universités lorsqu'il est ainsi transféré aux diplômés.

9 Alors même que des acteurs publics cherchent des solutions de court terme à la viabilité parfois douteuse (comme la privatisation de la loterie envisagée en Illinois ou sa concession à un contractant privé dans l'Indiana), les étudiants québécois ont le mérite d'apporter des éléments de réflexion dans la recherche d'une solution durable pour l'enseignement supérieur.

\section{NOTES}

1. $42,8 \%$ contre $76,4 \%$ pour l'ensemble des pays de l'OCDE ( $81,3 \%$ pour la France) : OCDE, Regards sur l'éducation 2006.

2. College Board, Trends in College Pricing 2007. http://www.collegeboard.com/press/ releases/189547.html.

3. A. Usher et A. Cervenan, Global higher Education Rankings 2005, Educational Policy Institute.

4. Pour un aperçu de ce débat, voir Peter Tulip, Financing Higher Education in the United States, Economics Department, 12/2007.

5. Valérie Vierstraete, Les frais de scolarité, l'aide financière aux études et la fréquentation des établissements d'enseignement postsecondaires : comparaison à l'échelle internationale et étude de scénarios pour le Québec, juin 2007, étude menée pour le MELS. 
6. La fréquentation avait reculé de 6,7 \% après que les droits de scolarité ont triplé au début des années 1990, op. cit.

7. FEUQ, Mémoire pour un nouveau pacte en éducation universitaire, novembre 2007.

INDEX

Index géographique : États-Unis, Québec, Canada

Mots-clés : droits de scolarité, enseignement supérieur, université

\section{AUTEUR}

\section{MAROUSSIA RAVEAUD}

Maître de conférences, Université du Mans. 